\title{
Débits écologiques : la place des modèles d'habitat hydraulique dans une démarche intégrée
}

\section{Ecological flows: the role of hydraulic habitat models within an integrated framework.}

\author{
N. Lamouroux ${ }^{1}$, B. Augeard ${ }^{2}$, P. Baran ${ }^{3}$, H. Capra ${ }^{1}$, Y. Le Coarer ${ }^{4}$,

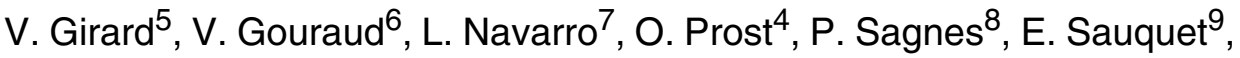 \\ L. Tissot $^{6}$ \\ 1 IRSTEA, UR MALY, 5 rue de la Doua, CS 70077, 69626 Villeurbanne Cedex, France \\ 2 ONEMA, 5 allée Félix Nadar, 94300 Vincennes, France \\ 3 ECOGEA, 352 avenue Roger Tissandié, 31600 Muret, France \\ IRSTEA, UR HYAX, 3275 route de Cézanne, CS 40061, 13182 Aix-en-Provence Cedex 5, France \\ 5 Asconit-Consultants, Parc Scientifique Tony Garnier, 6-8 espace Henry Vallée, 69366 Lyoncedex 07, France \\ 6 EDF Recherche et Développement, Laboratoire National d'Hydraulique et Environnement, 6 quai Watier, \\ 78401 Chatou Cedex, France \\ 7 Agence de l'eau Rhône Méditerranée et Corse, 2 allée de Lodz, 69007 Lyon, France \\ 8 Onema, pôle écohydraulique, IMFT, Allée du Pr. C. Soula, 31400 Toulouse. \\ 9 IRSTEA, UR HHLY, 5 rue de la Doua, CS 70077, 69626 Villeurbanne Cedex, France
}

Résumé - Deux types d'approches techniques complémentaires sont utilisées pour guider l'établissement des débits écologiques, à l'échelle des tronçons de cours d'eau (ex. : débits réservés) comme à l'échelle de bassins versants (ex. : débits objectifs d'étiage). Les approches « hydrologiques » visent à quantifier les altérations de multiples caractéristiques du régime hydrologique et reposent sur l'identification (délicate) de relations empiriques entre altérations hydrologiques et biologiques. Les approches « habitat hydraulique », ciblées sur les débits bas à moyens, couplent des modèles hydrauliques et des modèles biologiques pour traduire certaines modifications hydrologiques en modification de qualité de l'habitat hydraulique pour les organismes. Elles ont parfois apporté des prédictions convaincantes des effets biologiques des modifications de débits d'étiage. Ces deux approches techniques ne fournissent pas directement de valeurs de débits écologiques. Nous formalisons ici une démarche technique de définition des débits écologiques, basée sur la comparaison de scénarios de gestion et une meilleure combinaison des deux approches. La démarche comprend quatre étapes : (1) la description du contexte hydrologique naturalisé et actuel, des usages actuels et des scénarios de gestion envisagés (2) la description du contexte écologique au sens large, (3) l'identification des métriques pertinentes (hydrologiques et/ou habitats et/ou autres) pour décrire les effets des scénarios (modifications des usages, effets sur le milieu) et (4) la comparaison des scénarios. Cette démarche ne se passe pas d'expertise et doit 
s'accompagner d'une définition des objectifs partagée par les acteurs ainsi que de retours d'expérience, aspects non détaillés ici.

Mots-clés - débits environnementaux, débit réservé, prélèvements d'eau, hydroélectricité, microhabitat

\begin{abstract}
Two complementary technical approaches are used worldwide to guide the definition of e-flows, at the scale of stream reaches and/or stream catchments. Hydrological approaches are based on the description of the alteration of many components of the flow regime; they rely on the (difficult) identification of relationships between flow alteration and biological alteration. Hydraulic-habitat approaches essentially deal with low to medium flow rates. They combine hydraulic models of reaches with biological models in order to translate some hydrological changes into habitat changes for aquatic organisms. They sometimes provided convincing predictions of the biological effects of low flow changes. These two approaches do not directly provide e-flow values. Here, we formalize a technical framework for defining e-flows, based on the comparison of management scenarios and an improved combination of hydrological and habitat approaches. Four steps are involved: (1) the description of the natural and current hydrological contexts, current water uses and management scenarios; (2) the description of the ecological context; (3) the identification of appropriate metrics (based on hydrology or habitat) to describe the effects of scenarios (changes in water uses and ecological effects); (4) the comparison of scenarios. Applying this framework requires expertise, well defined objectives shared by stakeholders and appropriate monitoring of e-flow implementation.
\end{abstract}

Key words - environmental flows, instream flows, water abstraction, hydroelectricity, microhabitat

\section{INTRODUCTION : LA NOTION DE DÉBITS ÉCOLOGIQUES ET LES CONTEXTES RÉGLEMENTAIRES}

Les notions de débits « biologiques », " écologiques » ou " environnementaux » (e-flows en anglais) font l'objet de multiples définitions, souvent associées au contexte réglementaire considéré. Dans cet article, nous ne ferons pas de différence entre ces termes et adopterons une définition large d'un régime de « débits écologiques » : celle de la déclaration de Brisbane proclamée au 10th International River symposium and International Environmental Flows Conference à Brisbane, Australie (http://www.watercentre.org/news/ declaration, 2007). La déclaration décrit les débits écologiques comme la "quantité, la saisonnalité et la qualité des débits nécessaires à la durabilité des écosystèmes d'eau douce et estuariens ainsi qu'aux besoins et au bien-être des hommes qui dépendent de ces écosystèmes ». Cette définition large nous permettra de proposer une démarche de définition des régimes écologiques transposables à différents cadres réglementaires et schémas de gestion.

La question des débits écologiques concerne deux échelles spatiales principales, celle du tronçon de cours d'eau (défini ici comme une longueur de cours d'eau comportant plusieurs séquences de faciès géomorphologiques de type 
cascade, radier, plat et/ou mouille ...) et celle du réseau hydrographique dans sa totalité (bassin versant). À l'échelle des tronçons de cours d'eau, la plupart des applications relatives aux débits relèvent des études d'impacts des ouvrages et prises d'eau. En France, la réglementation concernée est principalement celle des débits réservés à laisser à l'aval des ouvrages construits dans le lit d'un cours d'eau (article L21418 du Code de l'Environnement et circulaires associées; République française, 2011). À l'échelle des bassins versants, la question des débits écologiques prend une importance croissante à l'échelle internationale (Acreman et al., 2014b), du fait (1) du besoin de définir des standards (valeurs par défaut) de débits à maintenir dans les cours d'eau en l'absence d'étude plus détaillées (Poff et al., 2010 ; Snelder et al., 2011) et (2) de réglementations impliquant la définition de débits écologiques à l'échelle des bassins (exemple de la Directive Cadre Européenne sur l'Eau - DCE ; Acreman \& Ferguson, 2010). Un guide européen est récemment paru pour inciter à définir des débits écologiques dans les bassins versants (European Commission, 2015). En France, c'est en particulier au travers des SDAGE, des SAGE et/ou des projets de gestion quantitative des prélèvements (définitions de débits objectifs d'étiage, de volumes prélevables ; Lang Delus, 2011) qu'apparaît le besoin de définir des débits écologiques dans les bassins (ex. : Floury et al., 2013 ; Baran et al., 2015).

Dans cet article, nous décrivons succinctement les deux types d'approches techniques les plus utilisées dans le monde pour définir des débits écologiques, l'approche "hydrologique" et l'approche "habitat hydraulique", ainsi que les éléments scientifiques qui soustendent leur bonne utilisation. Nous formalisons ensuite une démarche technique générale pour établir ces débits écologiques, basée sur la comparaison de scénarios de gestion, applicable à l'échelle du tronçon comme à celle du réseau hydrographique. Si cette démarche concerne essentiellement les aspects écohydrologiques, elle permet néanmoins l'intégration d'aspects socio-économiques qui ne sont pas détaillés dans cet article (Pahl-Wostl et al., 2013). Nous recadrons la place des modèles d'habitat hydraulique, aujourd'hui fréquemment utilisés, dans cette démarche générale.

\section{L'APPROCHE «HYDROLOGIQUE »}

\subsection{Principe}

L'approche « hydrologique » consiste à quantifier les altérations du régime hydrologique, ici définies comme des modifications par rapport à une situation naturelle ou naturalisée (sans influence humaine), sur la base desquelles sont identifiées des limites d'altération « acceptables » d'un point de vue écologique. Les premiers développements de ce type d'approche ont concerné quelques statistiques de débit particulièrement concernées par les usages, comme le débit minimum (débit instantané à maintenir dans le cours d'eau à l'aval des ouvrages). Les approches utilisées aujourd'hui sont bien plus complètes et s'intéressent à l'ensemble des caractéristiques du 
régime hydrologique importantes pour les milieux : (1) étiages, crues et variations rapides ; (2) intensité, fréquence, durée et saisonnalité des évènements (Richter et al., 1996, 1997 ; Poff et al., 2010). Différents logiciels sont disponibles pour calculer les altérations hydrologiques liées aux activités humaines (ex. : logiciel IHA du Nature Conservancy, Richter et al., 1996 ; indicateurs d'éclusées, Courret, 2015). L'exemple de la Figure 1 comporte la liste de 33 statistiques de débits journaliers fréquemment utilisées pour décrire les altérations de régime hydrologique.

\subsection{Bases scientifiques et validations biologiques}

L'utilisation d'une approche « hydrologique " suppose d'identifier des relations quantitatives entre les altérations hydrologiques et leurs effets biologiques (relations débits-écologie, "flowecology relationships"), à partir desquelles il faudrait identifier des seuils d'altération de débits acceptables. La Figure 1 montre un exemple de relation débits-écologie, qui relie la couverture végétale des plaines alluviales à la durée totale des crues dans l'année. Identifier de telles relations reste délicat, ce qui explique la difficulté de mise en œuvre de l'approche hydrologique. Il existe de très nombreuses études de cas qui montrent que l'altération de nombreux aspects du régime peut avoir d'importants effets écologiques (Poff et al., 1997, 2010). Ainsi, de façon globale, l'existence d'effets écologiques induits par l'altération des débits ne peut être remise en cause. Néanmoins, les lois quantitatives générales qui pourraient refléter ces effets sont difficiles à identifier (Murchie et al. 2008, Poff \& Zimmerman, 2010, Webb et al., 2015). De nombreuses études s'accordent sur des effets écologiques négatifs (indiqués par des métriques d'abondance et de diversité) de réductions de débits d'étiage et de crue de plusieurs dizaines de \% (Poff \& Zimmerman, 2010 ; Carlisle et al., 2011; Rolls et al., 2012). De même, l'effet négatif des crues pendant les périodes de reproduction ou de post-émergence sur le recrutement des poissons est bien documenté (Capra et al., 2003 ; Cattanéo, 2005a, 2005b). Mais plus généralement, tous les aspects du régime hydrologique n'ont pas nécessairement la même importance dans un contexte donné (Jowett \& Biggs, 2006 ; Snelder \& Lamouroux, 2010 ; Gido et al., 2013), et les effets écologiques des altérations de régime peuvent dépendre d'autres aspects environnementaux comme la qualité de l'eau, la morphologie ou les interactions biotiques.

Les expériences passées de restauration du régime hydrologique ont également fait l'objet de synthèses récentes (Olden et al., 2014 ; Gillepsie et al., 2015). Les succès sont rapportés dans environ la moitié des études de cas, mais les mesures de restauration et les stratégies d'évaluation sont trop variées pour en déduire des enseignements généraux sur les mesures de restauration les plus efficaces. Ce type de synthèse met en évidence les limites des suivis écologiques des opérations de restauration, qui s'inscrivent rarement sur le long-terme et manquent de descriptions précises des états initiaux des communautés aquatiques (Bernhardt et al., 2005 ; Souchon et al., 2008). La 


\section{Approche « hydrologique "}

\section{Calculs d'indices d'altération hydrologique}

(d'après Richter et al., 1996)

\begin{tabular}{|lll|}
\hline \multicolumn{3}{|c|}{ Exemple de 33 indicat eurs de débit } \\
Moy_Octobre & Moy_Septembre & Nb_Jour_assec \\
Moy_Novembre Mini_1_jour & Indice_débit_base \\
Moy_Décembre Mini_3_jours & Date_minimum \\
Moy_Janvier & Mini_7_jours & Date_maximum \\
Moy_Février & Mini_30 _jours & Nb._périodes_ét iage \\
Moy_Mars & Mini_90_jours & Durēe_périodés_étiage \\
Moy_Avril & Maxi_1_jour & Nb._crues \\
Moy_Mai & Maxi_3_jours & Durée_crues \\
Moy_Juin & Maxi_7_jours & Vitesse_mont ées \\
Moy_Juillet & Maxi_30_jours & Vitesse_descentes \\
Moy_Août & Maxi_90_jours & Nb._inversions \\
\hline
\end{tabular}

\section{Relations empiriques " débits-écologie »}

(d'après Webb et al., 2015)

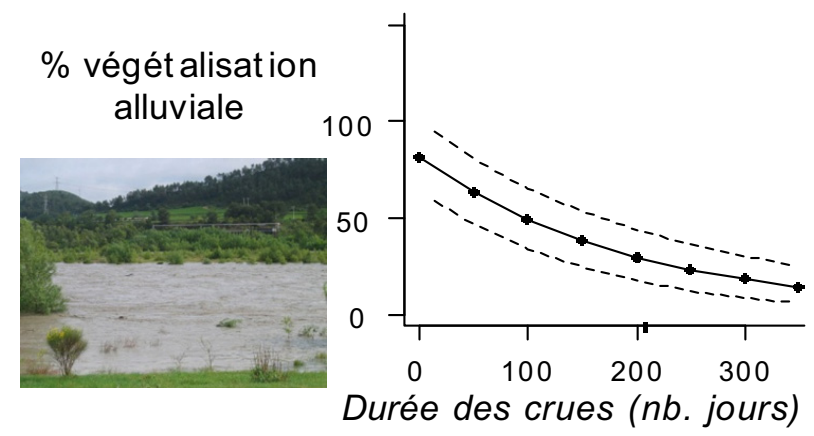

Fig. 1. Représentation simplifiée de l'approche « hydrologique " pour établir des débits écologiques. L'approche consiste à quantifier l'altération du régime de débits par rapport à une situation naturalisée, en utilisant de nombreux descripteurs de l'ensemble des aspects du régime (débits mensuels, amplitudes, durées et saisonnalité des débits extrêmes, vitesses de variation ...). Par ailleurs, des relations empiriques "débits-écologie " sont recherchées, ici entre la durée des crues et la végétalisation de la plaine alluviale.

Fig. 1. Principles of hydrologic approaches used to define e-flows. Hydrological approaches combine the estimation of many characteristics of flow alteration (relative to an unregulated situation) with empirical "flow-ecology" relationships. The example shown here relates the extent of alluvial vegetation in the floodplain to the annual flood duration.

description des états initiaux se base fréquemment sur une seule année (Morandi et al., 2014), ne permettant pas de prendre en compte la variabilité inter-annuelle. Cela rend l'étude statistique des impacts difficile sauf changement drastique du système (Vaudor et al., 2015). 


\subsection{Utilisation}

Les premières approches « hydrologiques » (Tennant, 1976), basées sur une expertise physique et biologique de cours d'eau Nord-Américains, ont influencé la réglementation des débits réservés en France (débits à maintenir à l'aval des ouvrages). Cette réglementation définit des débits planchers exprimés en pourcentage du débit moyen interannuel (le module), au-dessus desquels un régime de débits réservés doit être défini. Aujourd'hui, parmi les approches existantes, la démarche ELOHA (Ecological Limits Of Hydrological Alteration; Poff et al., 2010) apparait comme la plus aboutie et la plus utilisée pour définir des débits écologiques standards à l'échelle des bassins. La démarche ELOHA fait partie des méthodes dites " holistiques " (Linnansaari et al., 2013), qui vont plus loin qu'une approche purement "hydrologique", considèrent d'autres aspects comme les aspects sociaux et peuvent intégrer des éléments d'habitat hydraulique. Néanmoins, la base technique d'ELOHA est une approche " hydrologique » détaillée qui comprend une description de l'ensemble des caractéristiques hydrologiques naturalisées des tronçons de cours d'eau concernés, une typologie de ces régimes hydrologiques naturalisés, une estimation de leurs altérations, puis une recherche de relations empiriques débits-écologie par types de tronçons. Les difficultés de mise en œuvre apparaissent le plus souvent à cette dernière étape (Kendy et al., 2012, McManamay et al. 2013), du fait des difficultés de validation biologique évoquées dans la section 2.2.
C'est pourquoi les applications d'ELOHA ont souvent concerné des études de bassins à enjeux forts, riches en données physiques et biologiques (Kendy et al., 2012).

En l'absence de règles quantitatives générales, l'approche « hydrologique " implique un degré d'expertise important pour identifier les limites acceptables d'altération. Un exemple illustratif est celui de la définition de débits écologiques compatibles avec les objectifs de la DCE au Royaume Uni, qui est issue de concertations entre experts (Acreman \& Ferguson, 2010). L'octroi de licences de prélèvements d'eau ou d'exploitation d'ouvrages (quel qu'en soit l'usage) considère comme indicateur d'altération des débits les écarts entre courbes de débits classés influencées et naturalisées (Environmental Flow Index ; Environment Agency, 2013). Les débits classés concernés sont des quantiles de débit journalier (par exemple Q95 pour les bas débits, le débit journalier dépassé $95 \%$ du temps). Un écart de plus de $10 \%$ (pour des quantiles de bas débits dans des tronçons sensibles comme les zones de reproduction du saumon) ou de plus de $30 \%$ (pour des quantiles de haut débit dans des tronçons moins sensibles) indique un risque pour l'état écologique des cours d'eau. Des volumes prélevables sont estimés dans les bassins versants sur la base de ces limites d'altérations hydrologiques. Néanmoins, la base scientifique de ces seuils d'altération utilisés au Royaume Uni est fragile, et ces seuils d'experts ne peuvent être considérés comme des généralités à appliquer strictement dans tous les contextes et à tous les usages. 


\section{L'APPROCHE « HABITAT HYDRAULIQUE »}

\subsection{Principe}

L'approche " habitat hydraulique » (Fig. 2) utilise des modèles d'habitat hydraulique pour traduire certaines modifications hydrologiques (généralement la modification des débits bas à moyens) en modifications hydrauliques (ex. : modifications de vitesses du courant et de hauteurs d'eau) puis en modification de qualité de l'habitat hydraulique pour les organismes (le plus souvent les poissons et les macroinvertébrés). Elle part du principe que le débit n'est pas le descripteur le plus pertinent de l'habitat des organismes aquatiques, qui évoluent dans des champs de vitesses, de hauteurs d'eau, de contraintes exercées sur le fond du lit ou d'autres caractéristiques hydrauliques du « microhabitat » (échelle spatiale assez fine pour refléter l'environnement instantané des organismes). La traduction hydraulique des modifications de débits est donc importante, car un même débit sera associé à des caractéristiques hydrauliques très différentes selon les cours d'eau en fonction de leur morphologie (ex. : pente, substrat, section en travers). L'approche part également du principe que les " préférences " des organismes pour leur habitat hydraulique dépendent de l'espèce, de son activité et de son stade de développement, tout en gardant à l'esprit que les caractéristiques hydrauliques ne sont pas suffisantes à elles seules pour décrire l'habitat des organismes (Fig. 3). Ainsi, il est important de quantifier la sensibilité aux modifications hydrauliques des communautés en place.

Les modèles d'habitat hydraulique couplent un modèle hydraulique qui décrit les caractéristiques hydrauliques des microhabitats (vitesse, hauteur d'eau...), avec des modèles de préférence des espèces et/ou stades de vie et/ou groupes d'espèces pour ces caractéristiques (Fig. 2). Utilisés le plus souvent à l'échelle des tronçons de cours d'eau, ces modèles d'habitat permettent de cartographier des valeurs d'habitat (souvent normalisées sous forme de scores de préférence variant entre 0 et 1) qui reflètent la qualité de l'habitat hydraulique pour les espèces considérées (Fig. 2). D'autres variables physiques comme le substrat ou la température sont parfois intégrées. Une variation de la valeur d'habitat moyenne sur le tronçon ou d'une " surface pondérée utile » SPU (produit de la valeur d'habitat et de la surface du tronçon) peut alors synthétiser l'impact d'une variation de débit sur la qualité de l'habitat hydraulique.

Les modèles d'habitat disponibles sont nombreux (Dunbar et al., 2012 ; Linnansaari et al., 2013). La plupart reposent sur un modèle hydraulique numérique (aujourd'hui souvent bidimensionnel) qui résout les équations de conservation de la masse et de l'énergie sur un tronçon de cours d'eau (par exemple : logiciel Phabsim, Bovee, 1982 ; River2D, Ghanem et al., 1996 ; en France : Evha, Ginot, 1998). Ces modèles nécessitent des données de topographie du lit en trois dimensions et une calibration hydraulique experte. D'autres modèles extrapolent des mesures hydrauliques faites à 


\section{Approche « habitat hydraulique »}

\section{Préférences biologiques (microhabitats)}

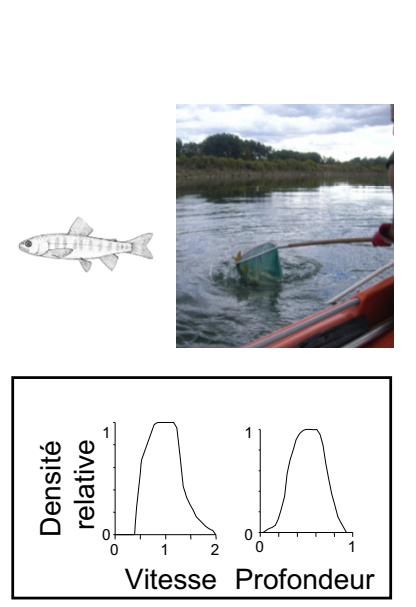

\author{
Couplage de modèles : hydraulique + préférences
}

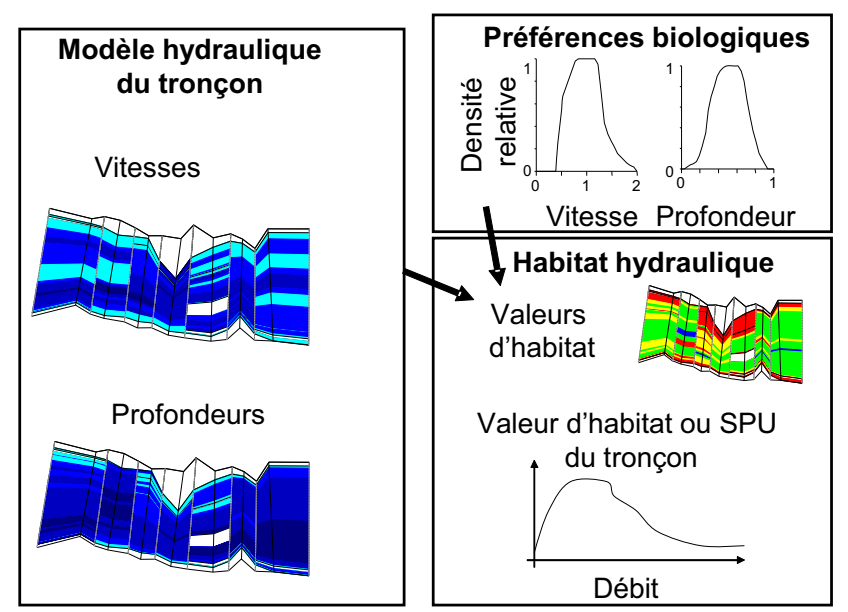

Fig. 2. Représentation simplifiée de l'approche « habitat hydraulique " pour établir des débits écologiques. L'approche se base sur l'existence de " préférences» des espèces aquatiques pour les caractéristiques hydrauliques de leur microhabitat. Ces préférences correspondent les plus souvent à des variations de densité relative (par rapport au maximum de densité) observées entre microhabitats. Un modèle d'habitat hydraulique couple un modèle hydraulique d'un tronçon de cours d'eau avec des modèles de préférence. Le modèle hydraulique permet de cartographier (par exemple) des vitesses et profondeurs à différents débits. Les modèles de préférence traduisent cette information hydraulique en cartes de valeurs d'habitat (généralement un score de préférence entre 0 et 1 ) pour les espèces considérées. Lorsque le débit varie, les variations de valeur d'habitat moyenne du tronçon (ou de surface utile SPU) quantifient les effets biologiques potentiels des variations de débit.

Fig. 2. Principles of hydraulic-habitat approaches used to define e-flows. Hydraulic-habitat models are based on observed "preferences" of aquatic species for the hydraulic characteristics of their microhabitats. Hydraulic-habitat models link a hydraulic model of a stream reach with biological preference models. The hydraulic model is used to map, e.g., velocities and water depths at various discharge rates. Preference models translate this information into habitat values (generally, preference scores between 0 and 1). When discharge changes, variations of the reach-averaged habitat value are used to quantify the potential biological effects of discharge variations.

3-4 débits de calage (par exemple en France : Lammi, Sabaton et al., 1995).

Des modèles d'habitat statistiques (par différence avec les modèles d'habitat numériques) reposent sur une modélisation directe des distributions statistiques des variables hydrauliques ponctuelles dans les tronçons de cours d'eau (histogrammes des vitesses, des hauteurs d'eau, des forces ponctuelles ; ex. : logiciel Stathab ; Girard et al., 2014a) ou des valeurs d'habitat du tronçon (logiciel Estimhab ; Lamouroux \& Capra, 2002 ; Lamouroux \& Souchon, 2002). Les modèles statistiques ne permettent pas une cartographie des valeurs d'habitat, et ne s'appliquent pas dans des morphologies fortement 


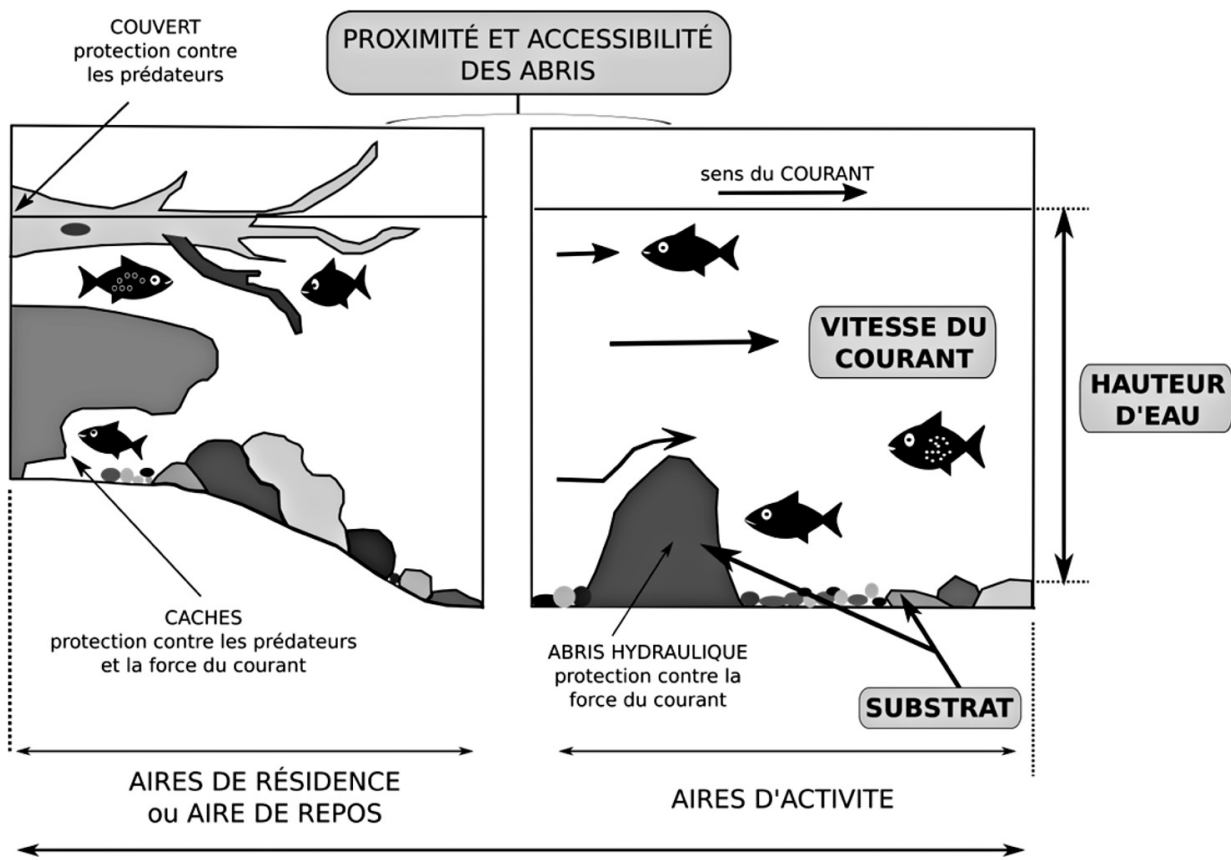

DOMAINE VITAL QUOTIDIEN

Fig. 3. Illustration de l'habitat physique quotidien en rivière (d'après Thévenet, 1998). Les individus utilisent des combinaisons de variables physiques (vitesses du courant, force, hauteur d'eau, substrat, abris, température ...) qui peuvent varier suivant leur activité et au cours de leur développement. Les modèles de préférence sont une simplification de ces comportements individuels.

Fig. 3. The physical habitat of organisms in rivers (from Thévenet, 1998). Aquatic organisms use combinations of several physical variables (e.g. flow velocities, shear stresses, water depth, substrate size, cover, temperature) that vary with life stages and biological activities. Hydraulic preference models are simplifications of these individual behaviors.

altérées (ex. : par chenalisation, recalibrage). En revanche, ils sont plus simples à mettre en œuvre que les modèles numériques, car leurs variables d'entrée principales sont les caractéristiques moyennes des tronçons de cours d'eau (débit, largeur, hauteur, taille granulométrique du substrat) mesurées à deux débits distincts. Ainsi, lorsqu'une cartographie des valeurs d'habitat n'est pas nécessaire, ils facilitent grandement la modélisation de l'habitat hydraulique. Ils permettent également des applications multi-sites et des simulations sur l'ensemble du réseau hydrographique d'un bassin versant (ex. : Snelder et al., 2011).

\subsection{Bases scientifiques et validations biologiques}

L'existence de préférences hydrauliques des poissons et des invertébrés d'eau courante à l'échelle du microhabitat fait l'objet d'une littérature 
abondante. Ces préférences résultent de processus multiples comme les besoins physiologiques, les interactions entre individus, ou les compromis entre ressources trophiques, capacité de nage et résistance au courant. Les préférences sont nécessairement une vision moyenne simplifiée des comportements individuels, qui varient en fonction de l'espèce, du stade de développement, de la taille et de la fonction vitale réalisée (alimentation, repos, reproduction; cf. Fig. 3). Les préférences d'un taxon peuvent également varier entre cours d'eau, par exemple lorsqu'une mauvaise qualité chimique du cours d'eau amène le taxon à rechercher des habitats plus courants et plus oxygénés (Lancaster \& Downes, 2010).

Les préférences de très nombreux taxons aquatiques pour leur microhabitat hydraulique ont été maintenant comparées entre de nombreux cours d'eau et à différentes saisons (Lamouroux et al., 1999, 2013 ; Girard et al. 2014b). Ces comparaisons ont montré que la majorité des modèles de préférence sont des relations non linéaires qui reflètent de très fortes variations de densité d'individus en fonction de l'hydraulique. Elles ont également montré qu'un modèle de préférence se transfère d'une rivière à l'autre dans $\sim 60 \%$ des cas, degré de transférabilité moyen qui varie fortement entre taxons et entre cours d'eau (Fig. 4). Néanmoins, ce degré de transférabilité justifie de réaliser des simulations d'habitat sur un cours d'eau en utilisant des modèles de préférence construits sur d'autres cours d'eau. De la même façon, ceci justifie des modélisations d'habitat hydraulique étendues à l'échelle des bassins versants.
Lors des recherches de relations quantitatives générales entre modifications hydrologiques et modifications biologiques (cf. Sect. 2.2), les modèles d'habitat ont apporté des éléments de validation convaincants concernant l'impact des débits d'étiage sur les populations. Des différences d'habitat dans l'espace, entre sites situés à l'amont et à l'aval des barrages, expliquent une part importante des différences biologiques entre ces sites (Baran et al., 1995). Les modèles d'habitat ont par ailleurs été utilisés pour prédire, dans le temps, les effets biologiques de restauration de débits (Jowett \& Biggs, 2006 ; Sabaton et al., 2008 ; Bradford et al., 2011 ; Lamouroux et al. 2015). Lorsque les modifications hydrauliques liés à la restauration des débits étaient fortes (c'est le cas de tronçons du Rhône où des débits minimaux ont été multipliés par des facteurs de 5 à 10 ; Lamouroux \& Olivier, 2015 ; Mérigoux et al., 2015), les modèles ont permis de prédire avec précision des modifications d'abondance des espèces (poissons et invertébrés) ou de structure de communauté (ex. : proportions de groupes d'espèces multipliées par 2 ou 3). Les exemples du Rhône (Fig. 5) sont convaincants (plus particulièrement pour les poissons) car ils concernent de nombreuses espèces et sont basés sur des modèles construits avant restauration, pour la plupart sur des sites indépendants des sites restaurés. De façon cohérente, lorsque les changements d'habitat sont plus modérés (c'était souvent le cas lors de l'expérience dite de la cellule « débits réservés " concernant la truite commune ; Sabaton et al., 2008), les résultats sont moins tranchés. 

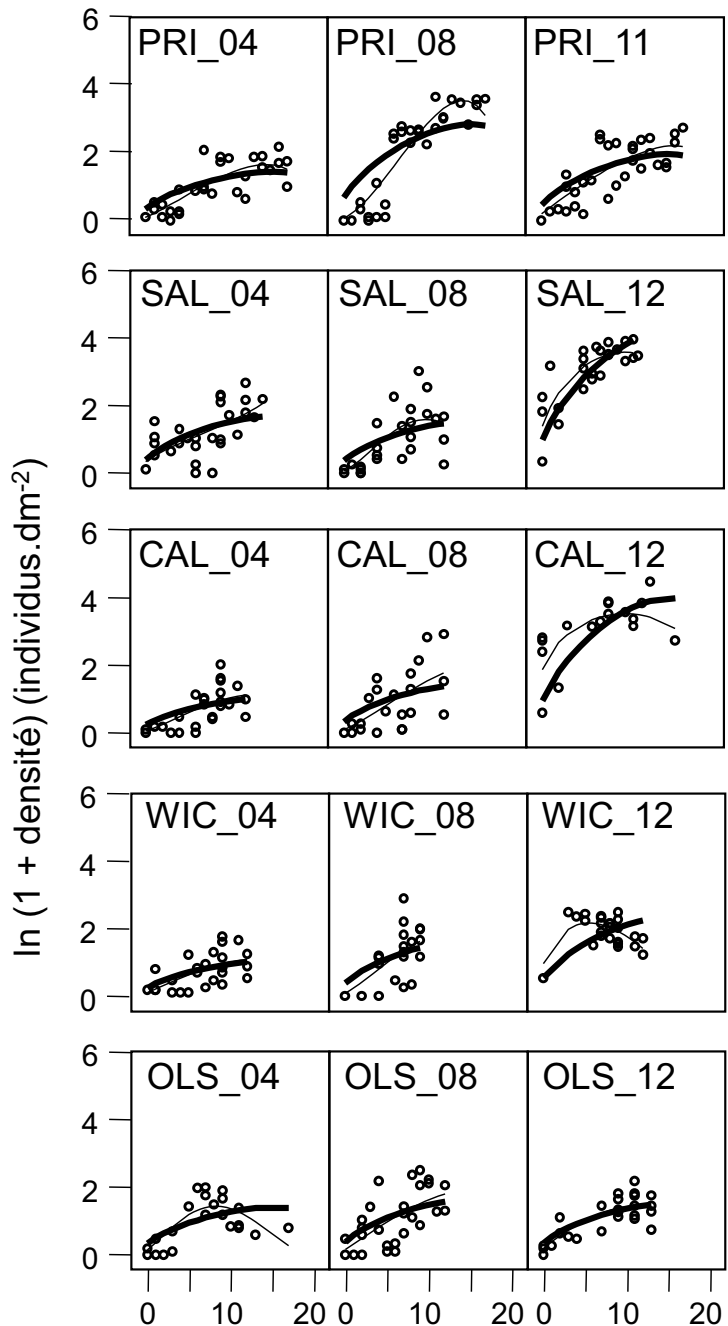

Contrainte au fond (hémisphère FST)

Fig. 4. Densité d'une larve d'éphémère (Baetis rhodani) en fonction de la contrainte de cisaillement sur le lit du cours d'eau (numéro d'hémisphère FST de Statzner \& Müller, 1989) dans des échantillons (chaque point correspond à un échantillon par Surbers) recueillis lors de 15 campagnes (rivière $\times$ date) réalisées dans 5 rivières allemandes à différentes dates. La ligne épaisse correspond à un modèle de préférence moyen entre campagnes, la ligne mince est un modèle spécifique à chaque campagne. Les étiquettes de chaque campagne combinent un code de cours d'eau (ex. : "PRI ») et le mois d'échantillonnage (ex. : « 04 », « 08 ») (d'après Lamouroux et al. 2010).

Fig. 4. Density of Baetis rhodani as a function of bed shear stress (FST hemisphere number; Statzner \& Müller, 1989) in Surber samples collected during 15 surveys (river $\times$ date) made in five rivers. The thick line is an average model across surveys. Thin lines are survey-specific models. Labels of surveys combine a river code (e.g. "PRl") and the sampling month (e.g. "04", "08") (from Lamouroux et al., 2010). 


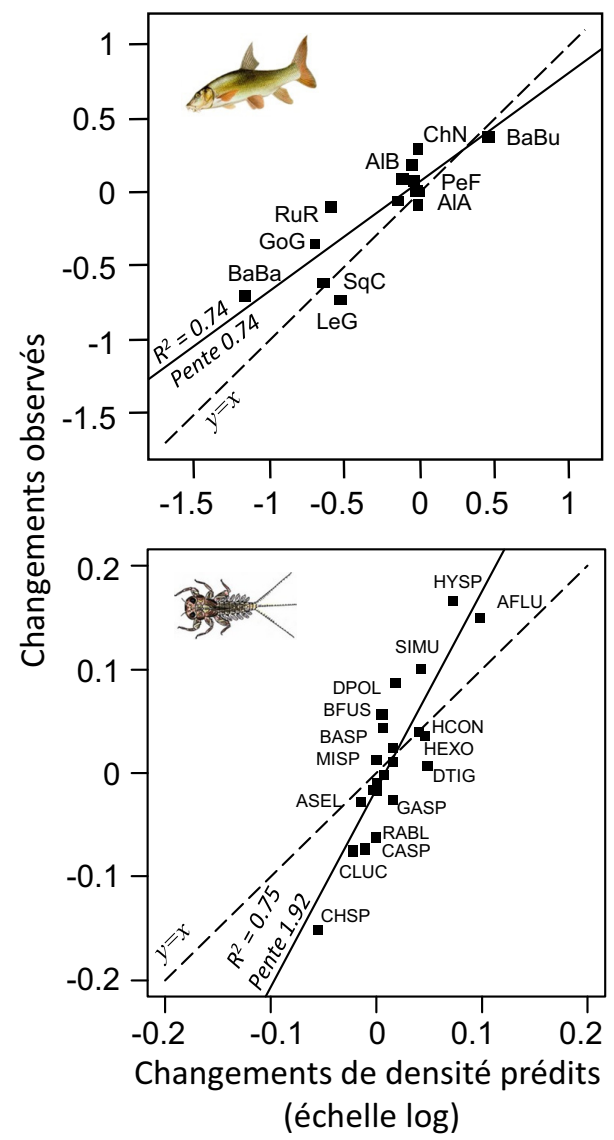

Fig. 5. Effets de l'augmentation de débits minimaux sur les densités de poissons et d'invertébrés : observations vs. prédictions par des modèles d'habitat hydrauliques. Ces exemples concernent le tronçon court-circuité du Rhône à Pierre-Bénite, où le débit est proche du débit minimal réglementaire pendant $\sim 300$ jours par an ; le débit minimum a été multiplié par 10 après restauration. Les étiquettes correspondent à différentes espèces de poissons et différents taxons invertébrés. D'après Lamouroux \& Olivier (2015), Mérigoux et al. (2015).

Fig. 5. Effects of flow restoration (minimum flow increases) on densities of fish and macroinvertebrate taxa: observations vs. predictions from hydraulic habitat models. These tests were made in a large regulated river where minimum flow is frequent ( 300 days per year) and was increased 10-fold. Labels correspond to different fish and invertebrate taxa. From Lamouroux \& Olivier (2015), Mérigoux et al. (2015).

\subsection{Utilisation}

Les modèles "d'habitat hydraulique" sont, avec les approches " hydrologiques ", les outils les plus utilisés dans le monde pour définir des débits écologiques à l'échelle des tronçons de cours d'eau (Tharme, 2003). Néanmoins, ils ont été critiqués depuis leur origine jusqu'à nos jours (Lamouroux et al. 
2010 ; Lancaster \& Downes 2010) pour être soit trop simplistes par rapport à la complexité de l'utilisation de l'habitat, soit trop complexes par rapport à une approche purement "hydrologique". Les tests récents de transférabilité des préférences biologiques (cf. Sect. 3.2 ; Lamouroux et al., 2013) précisent les limites de validité des modèles hydrauliques d'habitat et devraient réduire le champ de polémique. En France, les modèles d'habitat basés sur une modélisation hydraulique (Evha, http:// www.irstea.fr/dynam) ou simplifiée (Lammi, http://innovation.edf.com) ont été les plus utilisés dans les années 1980 à 2000. Aujourd'hui, les méthodes d'habitat statistiques (notamment le modèle Estimhab, http://www.irstea.fr/ dynam) sont les plus fréquemment utilisées. Des modèles statistiques de même type sont utilisés outre-mer (Saraeva \& Hardy, 2009 ; Snelder et al., 2011 ; Wilding et al., 2014).

La simplicité des variables d'entrée des modèles d'habitat statistiques facilite les applications sur plusieurs sites à l'échelle des bassins (exemple des études « volumes prélevables » du bassin Rhône-Méditerranée-Corse ; Floury et al., 2013). Des applications généralisées sur l'ensemble des tronçons d'un bassin sont également possibles, car les variables d'entrée peuvent être extrapolées spatialement (Lamouroux, 2008). Des simulations d'altération d'habitat généralisées apparaissent donc à l'échelle des bassins versants (Snelder et al., 2011 ; Miguel et al., 2016). Néanmoins, ces applications généralisées reposent sur des extrapolations spatiales et sont associées à une forte incertitude. En conséquence, elles fournissent des éléments de cadrage à l'échelle des bassins (pour la comparaison de scénarios de gestion et le repérage de zones particulièrement impactées) mais ne remplacent pas les études d'impact à l'échelle des tronçons de cours d'eau (Miguel et al., 2016).

\section{DEUX APPROCHES À COMBINER AVEC DE L'EXPERTISE}

La complémentarité des approches " hydrologique » et " habitat hydraulique ", et en particulier de leurs validations scientifiques, confirme que ces deux approches techniques gagnent à être combinées pour définir des débits écologiques. L'approche « hydrologique » est nécessaire pour identifier l'ensemble des modifications hydrologiques qui peuvent affecter les communautés biologiques. L'approche « habitat hydraulique » est plus ciblée sur les débits bas à moyens, et permet de traduire certaines modifications hydrologiques en modifications d'habitat, plus pertinentes car mieux reliées aux réponses biologiques par des validations de terrain. Le besoin de combiner ces deux types d'approche est largement reconnu dans la littérature internationale. Par exemple, l'apport potentiel des modèles d'habitat statistiques à l'échelle des bassins est mentionné dans les descriptions des approches "hydrologiques" les plus complètes (Poff et al., 2010 ; Wilding et al., 2014). En retour, les guides de modèles d'habitat rappellent l'importance d'intégrer la démarche dans une approche plus globale incluant une description des altérations hydrologiques et du contexte environnemental au sens 
large (Souchon et al., 2003). Le guide Européen récent sur les débits écologiques (European Commission, 2015), la législation française récente relative aux débits réservés (République Française, 2011) et les guides régionaux (Floury et al., 2013 ; Baran et al., 2015) vont également dans le sens d'une combinaison des approches.

Malgré ce large consensus, les approches " hydrologique » ont trop souvent été utilisées pour défendre l'idée d'un régime naturel "en miniature ", dont toutes les caractéristiques (intensité, fréquence et durée des évènements ...) seraient proportionnelles à celles du régime naturalisé. Aujourd'hui, les plus ardents défenseurs du régime naturel reviennent sur cette vision (Richter, 2010 ; Acreman et al., 2014a), peu réaliste d'un point de vue opérationnel et peu justifiée par l'état des connaissances sur les relations débits-écologie (Jowett \& Biggs, 2006). On retiendra néanmoins, dans la démarche de définition des débits écologiques, l'importance majeure de décrire l'ensemble des altérations hydrologiques jugées importantes pour les peuplements en place.

Les modèles d'habitat ont trop souvent été utilisés pour trouver des "chiffres magiques" (débit minimum biologique ou autre valeur réglementaire) issus de la lecture des courbes habitatdébit, ce qu'ils ne fournissent pas directement. II est donc important de mieux formaliser la démarche d'utilisation des modèles d'habitat, en la basant sur les validations biologiques existantes (Sect. 3.2). En particulier, on retiendra que les validations de modèles d'habitat ont essentiellement mis en évidence des liens entre modifications d'habitat et effets biologiques. La démarche que nous proposons se base donc sur l'analyse des différences d'habitat entre scénarios de gestion alternatifs.

La complexité des régimes hydrologiques, de leur altération par les usages, la diversité des milieux physiques et des réponses biologiques associées font qu'il n'y a pas de recette miracle (presse-bouton) pour identifier les débits écologiques. Dans cette situation, la démarche de mise en œuvre des approches « hydrologique » et « habitat hydraulique » devra être associée à une expertise à différentes étapes : identification des caractéristiques importantes du régime hydrologique, identification des groupes biologiques et espèces à considérer, analyse multicritères des impacts de scénarios de gestion sur les milieux et les usages. En conséquence, l'intervention d'experts formés et l'intégration des différentes parties prenantes dans la démarche sont fondamentales.

\section{UNE DÉMARCHE INTÉGRÉE CONSENSUELLE, EN QUATRE ÉTAPES}

Nous formalisons ici une démarche de comparaison technique de scénarios de gestion, qui vise à améliorer l'utilisation des outils disponibles pour la définition des débits écologiques (Fig. 6). Cette démarche fait appel à l'expertise, et doit s'accompagner d'une définition des objectifs partagés par les acteurs ainsi que de retours d'expérience, aspects non détaillés ici. La démarche que nous proposons emprunte des éléments d'approches 
1) Contexte hydrologique et scénarios

Chroniques de débits, débits classés et/ou statistiques de débit, naturalisés ou altérés par les usages actuels ou envisagés (scénarios)

\section{2) Contexte écologique général}

\section{Description du contexte biologique} (communautés, leur histoire, leur statut ...) et du contexte environnemental (qualité d'eau, thermie, connectivité ... )

\section{3) Identification de métriques pour décrire les effets des} scénarios (modifications des usages, effets sur le milieu)

Au vu des étapes précédentes, l'expert identifie les métriques pour décrire :

* les modifications hydrologiques (amplitudes, durées, saisonnalité ...) importantes pour les objectifs écologiques ; certaines seront traduites en modifications d'habitat hydraulique

* les modification des usages (disponibilité de la ressource ...)
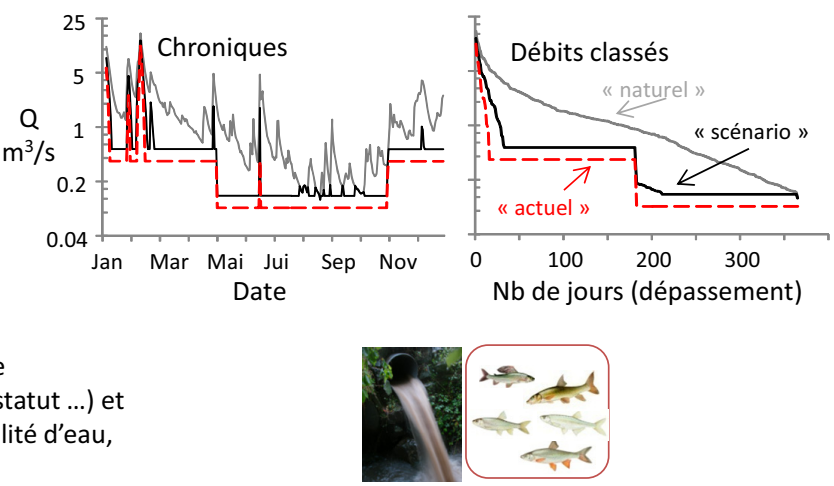

\section{4) Comparaison multicritères des scénarios de gestion}

Une décision de régime écologique est prise en comparant les variations de métriques entre scénarios

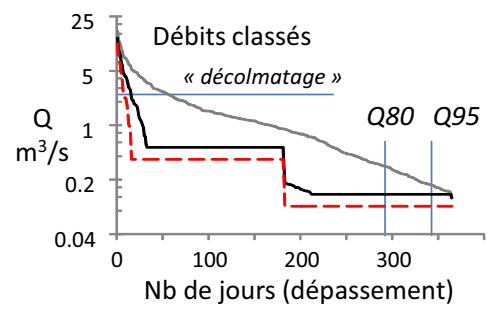

\begin{tabular}{|l|r|r|r|}
\hline Métrique & Naturel & Actuel & Scenario 1 \\
\hline Nb jour décolmatage & 55 & 8 & 15 \\
SPU Q95 chabot & $158 \mathrm{~m}^{2}$ & $-21 \%$ & $-6 \%$ \\
SPU Q80 chabot & $203 \mathrm{~m}^{2}$ & $-38 \%$ & $-27 \%$ \\
Q moyen utilisé & 0 & $1.09 \mathrm{~m}^{3} / \mathrm{s}$ & $0.88 \mathrm{~m}^{3} / \mathrm{s}$ \\
$\ldots$ & & & \\
\hline
\end{tabular}

Fig. 6. Une démarche consensuelle en quatre étapes pour l'établissement des débits écologiques. La démarche est illustrée par un exemple fictif. Le contexte hydrologique naturel, actuel et sous scénario (1) peut être décrit par des chroniques de débits ou des débits classés. Au vu du contexte écologique (2), des métriques sont définies pour décrire les modifications d'usage et de milieu entre scenarios (3). L'exemple donné retient la fréquence de décolmatage du lit et les valeurs des bas débits (quantiles Q80 et Q95), qui seront traduites en qualité d'habitat (SPU, surface utile pour le chabot). Ces métriques et les effets sur les usages (ici le débit utilisé) sont comparés entre scénarios pour guider la décision (4).

Fig. 6. A consensual approach in four steps for guiding e-flows definition, illustrated using a fictive example. The unregulated, current and future hydrological scenarios (1) are here described by discharge series and duration curves. After considering the general ecological context (2), metrics are identified for describing changes in water uses and ecological effects (3). In the example, the metrics retained indicate the frequency of a "flushing discharge" and the level of low flows (Q95 and Q80 quantiles), which will be translated into habitat suitable areas (SPU for a fish species). These metrics and the effect on water uses (here, the available discharge) are compared across scenarios (4).

dites « holistiques » (approches DRIFT, King et al., 2003 ; ELOHA, Poff et al., 2010). Néanmoins, notre démarche se veut plus simple que les approches holistiques existantes, dont la mise en œuvre est souvent complexe et/ou lourde. Nous visons une démarche applicable à la majorité des situations 
opérationnelles et cohérente avec les éléments de validation biologique des approches « hydrologique » et « habitat hydraulique $»$.

\section{1 Étape 1) Contexte hydrologique naturalisé et actuel, usages et scénarios de gestion}

Trop d'études ciblant la définition de débits écologiques se lancent dans des discussions de détail sans description suffisante de l'hydrologie actuelle et naturalisée (c'est-à-dire reconstituée sans influence des aménagements et prélèvements). II est fréquent (voire systématique si l'on travaille à l'échelle des bassins) d'avoir à extrapoler les caractéristiques du régime à partir de stations de jaugeage. Pour cela il existe plusieurs bases de données hydrologiques extrapolées (ex. : débits moyens et débits d'étiage naturalisés de Riffard et al., 2011 ; débits classés naturalisés de Sauquet et Catalogne, 2011, Pella et al., 2012). Une grande variété de modèles hydrologiques de bassins (Andréassian et al., 2006 ; Singh \& Frevert, 2006) ainsi que différentes méthodes de régionalisation (Blöschl et al., 2013) permettent également des extrapolations hydrologiques. II est utile de caractériser le fonctionnement hydrologique du bassin, notamment en matière d'alimentation en eau à l'étiage. Les extrapolations spatiales de statistiques de débits peuvent être assorties d'une incertitude forte, notamment en tête de bassin (Sauquet, 2006; Lamouroux et al., 2014). Il est donc important de quantifier l'incertitude des extrapolations hydrologiques. Dans de nombreux cas, un équipement hydrométrique des sites ou des jaugeages complémentaires (Catalogne et al., 2014) peuvent s'avérer nécessaire avant de mettre en œuvre une modélisation des aspects écologiques.

La description des usages actuels ou envisagés est importante dès cette étape, d'une part pour reconstituer l'hydrologie naturalisée et d'autre part pour identifier les caractéristiques du régime altérées par les usages. Par exemple, une prise d'eau hydroélectrique générera, le plus souvent, des débits minimum de durée bien plus longue qu'une prise d'eau d'irrigation. De surcroît, les ouvrages hydroélectriques sont parfois arrêtés l'été, ce qui est rare pour l'irrigation. Ainsi, les caractéristiques choisies pour décrire les débits et leurs modifications dépendront des usages actuels et envisagés. Naturellement, les étapes suivantes de la démarche (descriptions du contexte écologique, du cycle de vie des espèces, quantification des effets ...) amèneront probablement à repenser et adapter les scénarios. Ainsi, les étapes de la démarche proposées ici pourront faire l'objet d'itérations successives.

Autant que possible, la description hydrologique devra concerner tous les aspects du régime qui peuvent avoir des effets écologiques (étiages, crues et variations rapides des débits; intensité, fréquence, durée et saisonnalité des évènements ; courbes de tarissement). Parmi les nombreuses variables permettant de décrire les régimes hydrologiques, les chroniques de débits journaliers et les courbes de débits classés sont particulièrement utiles dès lors qu'elles sont disponibles. Sur l'exemple fictif de la Figure 6, elles permettent d'identifier les caractéristiques 
hydrologiques les plus altérées (dans cet exemple, les débits bas à moyens, les débits de hautes eaux). Ces descriptions peuvent être déclinées pour des années sèches ou "moyennes ", et peuvent être résumées par des statistiques annuelles ou interannuelles comme les VCN (débits minimaux sur $\mathrm{N}$ jours consécutifs) ou des débits de crue d'une fréquence donnée. Les moyennes mensuelles peuvent également être utiles mais n'ont pas toujours de sens physique dans les cas où les variations infra-mensuelles sont fortes (exemple des DOM ou des cours d'eau de montagne). Pour certains usages comme la gestion en éclusées hydroélectriques, il sera important d'utiliser des descriptifs des variations infra-journalières (Courret, 2015). Enfin, dans les cours d'eau naturellement intermittents, la quantification des assecs dans l'espace et dans le temps est un préalable à l'analyse (Snelder et al., 2013 ; Pelte et al., 2014).

Lorsque les débits journaliers sont disponibles, il existe des supports logiciels associés aux approches " hydrologiques » pour caractériser les altérations hydrologiques (exemples des indicateurs d'altération hydrologique de la Figure 1, logiciel IHA, Richter et al., 1996 ; d'autres sont recensés par Rinaldi et al., 2013). Ces logiciels ont l'avantage de quantifier les altérations, bien qu'ils fournissent de très nombreuses statistiques dont le lien avec l'écologie n'est pas toujours garanti. Ces approches ne font pas l'objet de support logiciel en français à l'heure actuelle. La pratique française consiste plutôt à identifier par l'expertise les caractéristiques hydrologiques principalement influencées par les ouvrages et dont les effets écologiques sont jugés importants.

Pour les applications à l'échelle des bassins versants, la démarche ELOHA recommande à cette étape de réaliser une typologie de cours d'eau en fonction de leur hydrologie et leur morphologie (ex. : pente ou géologie). De telles classifications (ex. : Snelder et al., 2009) peuvent être utiles pour aborder le problème de gestion et identifier des sites représentatifs sur lesquels la modélisation des impacts sera affinée. Dans notre démarche, nous ne donnons pas de directive particulière pour simplifier le problème à l'échelle des bassins versants, pour deux raisons. Tout d'abord, suivant les objectifs de gestion, une typologie des tronçons du bassin pourra se baser sur différents critères: altérations hydrologiques mais aussi enjeux écologiques, enjeux de conservation, représentativité des cours d'eau, enjeux socio-économiques... Ainsi la réalisation éventuelle d'une typologie doit rester aussi flexible que possible. Ensuite, les débits journaliers ne sont pas toujours disponibles de façon distribuée sur l'ensemble des tronçons du bassin, rendant l'approche parfois délicate à mettre en œuvre.

\section{2 Étape 2) Le contexte écologique général}

Une description générale du contexte biologique et environnemental au sens large est nécessaire pour identifier l'ensemble des enjeux, pour décrire des facteurs qui peuvent interagir avec l'habitat physique et/ou apparaître plus limitants, et pour mieux peser le risque que représentent des altérations hydrologiques ou d'habitat. 
La description du contexte biologique comprend entre autres la liste des espèces en place (poissons, macroinvertébrés, écrevisses, autres) dans le périmètre de l'étude et autour, leur histoire récente et à plus long terme, leur statut de protection et les enjeux de conservation, leur caractère invasif, les interactions biotiques majeures, le rempoissonnement. Les cycles de vie des espèces pourront être décrits afin d'identifier les stades de développement et les saisons les plus sensibles, pendant lesquels une attention particulière devra être donnée aux altérations hydrologiques.

La description du contexte environnemental couvre notamment des aspects physico-chimiques, thermiques et hydromorphologiques (Gouraud et al., 2008). Par exemple, l'impact négatif d'une réduction des débits d'étiage peut être renforcé par la concentration de polluants et un réchauffement important. Les altérations des échanges avec la nappe peuvent altérer les habitats de reproduction et modifier les conditions thermiques. Les altérations de connectivité longitudinale peuvent empêcher la présence d'une espèce, sa recolonisation à partir du voisinage, et nuire à son développement quelle que soit la nature de l'habitat hydraulique. Les déséquilibres sédimentaires à l'échelle du bassin peuvent modifier la diversité des habitats au sein des tronçons. L'artificialisation du chenal peut augmenter l'impact négatif des hauts débits, pendant lesquels l'accès aux refuges (sous-berges, végétation rivulaire, affluents) serait important. L'altération des frayères et les accès aux zones de reproduction, dont la prise en compte par les modèles d'habitat est très partielle, peuvent s'avérer limitants pour les populations. À l'inverse, des apports d'affluents et des résurgences peuvent permettre une reconstitution rapide du débit le long du tronçon impacté et limiter les altérations d'échanges avec la nappe. Plus généralement, la configuration du site et les longueurs impactées sont des composantes à prendre en compte.

À cette étape il est utile de s'appuyer sur des études locales et les experts locaux (ex. : agents de l'Onema, animateurs de bassin versant, de SAGE ...). Des éléments de contexte sont également disponibles à l'échelle nationale, comme les états des lieux établis par les comités de bassin pour la DCE, les documents d'objectifs Natura 2000, l'Atlas du risque d'altération hydromorphologique Syrah (Valette et al., 2012), la base de données physiques du réseau hydrographique théorique (RHT) (Pella et al., 2012) ou des typologies d'ouvrages (Fahrner, 2010). Pour les études à l'échelle des bassins, cette étape de description du contexte écologique est particulièrement importante pour préciser les enjeux de gestion et/ou pour choisir des sites représentatifs du bassin sur lesquels la réflexion sur les débits écologiques pourra être plus poussée (Floury et al., 2013).

\section{3 Étape 3) Identification des métriques pertinentes pour décrire les effets des scénarios (modifications des usages, effets sur le milieu)}

Identifier les métriques pertinentes pour représenter les modifications 
d'usage et de milieu est l'étape la plus délicate, car elle fait appel à une expertise importante (Acreman \& Ferguson, 2010). Au vu des altérations hydrologiques actuelles et envisagées (étape 1), du contexte écologique général (étape 2), il s'agit d'identifier les métriques pertinentes pour comparer les situations naturalisées, actuelles, et différents scénarios de gestion. En ce qui concerne les usages, on se tournera naturellement vers les gains/pertes en termes de disponibilité de la ressource (exemple : nombre de jours associés à une restriction d'usage) et les coûts économiques associés aux différents scénarios. En ce qui concerne les effets sur le milieu, il s'agit de caractériser les modifications hydrologiques majeures, éventuellement saisonnalisées, en termes d'intensité mais aussi de durée (de crue, d'étiage ...), qui peuvent influencer le fonctionnement écologique du système. Certaines de ces modifications pourront être traduites en termes hydrauliques ou en modifications d'habitat pour les espèces et stades considérés (en particulier pour les débits bas à moyens). C'est donc à cette étape que l'approche combine des modifications hydrologiques et des modifications d'habitat hydraulique.

Dans l'exemple fictif de la Figure 6, il est intéressant de retenir des métriques qui reflètent les altérations des débits de hautes eaux et des débits bas à moyens, car les autres aspects du régime (débits extrêmes) sont moins altérés. Pour les débits de hautes eaux, une évaluation d'un débit permettant le décolmatage du substrat permet par exemple d'évaluer le nombre de jours de décolmatage par an. Pour décrire les débits bas à moyens, le Q95 et le Q80 peuvent être choisis. On peut utiliser un modèle d'habitat pour estimer les surfaces favorables aux espèces en place à ces débits, pour les différents scénarios. Concernant les usages, on peut par exemple s'intéresser au débit moyen utilisé. Ainsi, de multiples choix de métriques sont possibles à cette étape (ex. : travail sur les courbes de débits classés ou les VCN, travail sur des courbes d'habitat classées), rendant irréaliste une solution " pressebouton " et incontournable un recours à l'expertise.

Le contexte écologique décrit à l'étape 2 influe sur l'étape 3 de plusieurs façons. II permet d'une part d'identifier les espèces, stades de développement ou groupes d'espèces pour lesquels des simulations d'habitat sont utiles. II permet d'autre part de hiérarchiser l'importance que l'on donnera aux différentes métriques (ex: prise en compte d'interactions entre pollutions et étiage renforçant les impacts écologiques ; existence de données historiques signalant une altération limitante dans la situation actuelle). De même, les enjeux et objectifs écologiques, qu'ils soient réglementaires ou patrimoniaux, participent également à définir et hiérarchiser les métriques retenues.

\section{4 Étape 4) Comparaison multicritères des scénarios de gestion}

Vient ensuite l'heure du bilan multicritères, au cours duquel les modifications de métriques (modifications des usages, effets sur le milieu) sont comparées entre scénarios. C'est sur cette base que l'expert propose un régime de 
débits répondant aux objectifs fixés. Dans notre exemple fictif de la Figure 6, le scénario de gestion envisagé est un scénario intermédiaire entre la situation actuelle et la situation naturalisée. Pour ce scénario, les pertes d'habitat au Q80 pour le chabot (pris comme exemple d'espèce considérée) relatives à la situation naturalisée seraient ramenées à $27 \%$, tandis que la situation actuelle représente une perte d'habitat relative de $38 \%$; le débit moyen utilisé serait réduit de $19 \%$ par rapport à la situation actuelle. Au vu des objectifs et de l'état des populations aquatiques, une décision éclairée et concertée pourra être prise.

Le référentiel de base pour les calculs d'altérations peut être une situation naturalisée, la situation actuelle ou une autre, en fonction des objectifs à atteindre. Dans cette démarche, le retour à une situation naturalisée n'est pas systématiquement à rechercher (voir Poff \& Matthews, 2013). Néanmoins, les situations naturalisées et actuelles sont le plus souvent des repères importants pour décrire les conditions physiques qui ont structuré les communautés en place et réfléchir sur les altérations hydrologiques dans une démarche de comparaison de scénarios.

\section{CONCLUSIONS : RÔLE ACTUEL ET FUTUR DES MODÉLES D'HABITAT}

Nous avons formalisé une démarche technique de définition des débits écologiques, basée sur la comparaison de scénarios de gestion et une meilleure combinaison des approches « hydrologique " et « habitat hydraulique ". Les modèles d'habitat ne fournissent donc pas seuls des valeurs de débits écologiques mais contribuent à les choisir. Au sein de la démarche générale de comparaison de scénarios de gestion, ils traduisent une partie des modifications de débits en modifications d'habitat, dont le lien avec la réponse biologique est mieux validé. Les modèles permettent de trouver des compromis intéressants entre préservation/restauration des milieux et usages de l'eau, car une modification de débit peut se traduire en modification d'habitat plus ou moins importante suivant la morphologie du cours d'eau et les espèces concernées. Néanmoins, l'application de modèles d'habitat a un coût. Leur degré de mobilisation augmente donc généralement avec 1) les enjeux écologiques, 2) le degré d'altération hydrologique, et 3 ) l'échelle d'étude (utilisation plus fréquente à l'échelle des tronçons).

La démarche proposée ici est nécessairement schématique, et doit être adaptée en fonction des enjeux et des moyens. Elle peut être enrichie par l'intégration d'aspects supplémentaires, hydrologiques ou socio-économiques. Elle peut être complétée par une organisation de la négociation entre acteurs (identification des objectifs, discussion autour d'un tableau multicritères ; King et al., 2003 ; Poff \& Matthews, 2013). De même, si la pratique française cible souvent la modélisation de l'habitat des poissons, la démarche est ouverte à d'autres groupes biologiques qui sont traditionnellement moins étudiés (macroinvertébrés, végétation aquatique et riveraine, amphibiens, oiseaux; Fig. 1 et Fig. 5). 
Le panel de modèles d'habitat qui font aujourd'hui l'objet d'un support logiciel en France (Evha, Lammi, Estimhab) évoluera. En particulier, le modèle numérique Evha ne fait plus l'objet de suivi et ne permet pas de modélisation numérique bidimensionnelle (2D) des écoulements. Or, les modélisations $2 \mathrm{D}$ sont particulièrement utiles dans certaines situations comme la prédiction des effets de restaurations physiques, les études comportementales ou la modélisation des impacts des éclusées (Capra etal., 2011; Hauer et al., 2014). Plusieurs pistes sont envisagées pour faire évoluer le panel de modèles d'habitat disponibles. Une première piste consiste à développer des interfaces de modélisation de l'habitat modulaires et évolutives pour permettre (1) de combiner au mieux différents types de modèles hydrauliques et biologiques disponibles, (2) d'intégrer facilement de nouveaux modèles. Ceci permettrait notamment de mettre en œuvre les modèles hydrauliques les plus à jour et/ou compatibles avec la pratique opérationnelle française, en intégrant des modèles 2D. Une seconde piste consiste à poursuivre les études des préférences hydrauliques des organismes, de leurs déterminants et de leurs variations, afin d'améliorer la pertinence biologique des modèles d'habitat et mieux préciser leurs limites. II serait utile d'élargir les groupes biologiques pris en compte et de développer des modèles adaptés aux situations complexes (exemple des éclusées).

Plus généralement, la démarche globale formalisée ici doit être considérée comme évolutive, car elle s'appuie sur des validations de terrain reliant les modifications d'hydrologie et d'habitat aux modifications biologiques. Améliorer notre connaissance des liens hydrologie-habitats-biologie reste la principale voie d'amélioration de la démarche. Cela nécessite des retours d'expérience des opérations de mise en œuvre de débits écologiques coordonnés et organisés sur le long terme (Lamouroux et al., 2015) et comprenant une description des états avant opération faite sur plusieurs années. Ceci nécessite également de poursuivre les études des liens entre l'habitat physique et la dynamique des populations (Capra et al., 2003 ; Shenton et al., 2012). Préciser la démarche à l'échelle des bassins versants nécessitera également de progresser sur la quantification des altérations hydrologiques à large échelle, aujourd'hui associée à de fortes incertitudes. Enfin, la multiplication d'études concernant la gestion quantitative à l'échelle des bassins et impliquant l'utilisation de modèles d'habitat devrait permettre de mieux cerner l'apport des modèles d'habitat à cette échelle.

\section{REMERCIEMENTS}

Merci à Francis Gayou, Rémi Oudin, Stéphane Stroffek et Dominique Ombredane pour leurs commentaires sur le manuscrit.

\section{RÉFÉRENCES}

Acreman M.C. \& Ferguson A., 2010. Environmental flows and European Water Framework Directive. Freshwater Biology 55 : 32-48. 
Acreman M., Arthington A.H., Colloff M.J., Couch C., Crossman N.D., Dyer F., Overton I., Pollino C.A., Stewardson M.J. \& Young W., 2014a. Environmental flows for natural, hybrid, and novel riverine ecosystems in a changing world. Frontiers in Ecology and the Environment 12 : 466-473.

Acreman M.C., Overton I.C., King J., Wood P., Cowx I.G., Dunbar M.J., Kendy E. \& Young W., 2014b. The changing role of ecohydrological science in guiding environmental flows. Hydrological Sciences Journal 59 : 433-450.

Andréassian V., Bergström S., Chahinian N., Duan Q., Gusev Y.M., Littlewood I., Mathevet T., Michel C., Montanari A., Moretti G., Moussa R., Nasonova O.N., O’Connor K.M., Paquet E., Perrin, C., Rousseau A., Schaake J., Wagener T. \& Xie Z., 2006. Catalogue of the models used in MOPEX 2004/2005. IAHS Publication 307 : 41-93.

Baran P., Delacoste M., Dauba F., Lascaux J.M. \& Belaud A., 1995. Effects of reduced flow on brown trout populations downstream dams in French Pyrenees. Regulated Rivers: Research and Management 10 : 347-361.

Baran P., Longuevergne L., Ombredane D., Dufour S. \& Dupont N., 2015. Débit Minimum Biologique (DMB) et gestion quantitative de la ressource en eau. Centre de Ressources et d'Expertise Scientifique sur l'Eau en Bretagne : $124 \mathrm{p}$.

Bernhardt E.S., Palmer M.A., Allan J.D., Alexander G., Barnas K., Brooks S., Carr J., Clayton S., Dahm C., FollstadShah J., Galat D., Gloss S., Goodwin P., Hart D., Hassett B., Jenkinson R., Katz S., Kondolf G.M., Lake P.S., Lave R., Meyer J.L., O'donnell T.K., Pagano L., Powell B. \& Sudduth E., 2005. Synthesizing U.S. river restoration efforts. Science 308 : 636-637.

Blöschl G., Sivapalan M., Wagener T., Viglione A. \& Savenije H., 2013. Runoff prediction in ungauged basins. Synthesis across processes, places and scales. Cambridge University Press, Cambridge, UK : $465 \mathrm{p}$.

Bovee K.D., 1982. A Guide to Stream Habitat Analysis Using the Instream Flow Incremental Methodology. Instream Flow Information Paper 12. U.S. Fish and Wildlife Service, Fort Collins, Colorado, USA : $248 \mathrm{p}$.

Bradford M.J., Higgins P.S., Korman J. \& Sneep J., 2011. Test of an environmental flow release in a British Columbia river: does more water mean more fish? Freshwater Biology 56 : 2119-2134.

Capra H., McNeil E., Bouillon M.-C., Pella H., Alfaro C., (2011) Intérêt d'un modèle hydrodynamique en deux dimensions pour interpréter le comportement des poissons dans les grands cours d'eau. La Houille Blanche 6 : 28-33.

Capra H., Sabaton C., Gouraud V., Souchon Y. \& Lim P., 2003. A population dynamics model and habitat simulation as a tool to predict brown trout demography in natural and bypassed stream reaches. River Research and Applications 19 : 551568.

Carlisle D.M., Wolock D.M \& Meador M.R., 2011. Alteration of streamflow magnitudes and potential ecological consequences: a multiregional assessment. Frontiers in Ecology and the Environment 9 : 264-270.

Catalogne C., Sauquet E. \& Lang M., 2014. Valorisation des données de jaugeages épisodiques pour l'estimation du débit de référence d'étiage QMNA5. La Houille Blanche 4 : 78-87.

Cattanéo F., 2005a. Does hydrology constrain the structure of fish assemblages in French streams? Local scale analysis. Archiv für Hydrobiologie 164 : 345365.

Cattanéo F., 2005b. Does hydrology constrain the structure of fish assemblages 
in French streams? Regional scale analysis. Archiv für Hydrobiologie 164 : 367385.

Courret D., 2015. Problématique des impacts de la gestion par éclusées des aménagements hydroélectriques sur les populations de poissons - Caractérisation des régimes d'éclusées et du niveau de perturbation hydrologique, et réflexions sur les mesures de mitigation. Thèse de Doctorat. INP-Toulouse, France : $218 \mathrm{p}$.

Dunbar M.J., Alfredsen K. \& Harby A., 2012. Hydraulic-habitat modelling for setting environmental river flow needs for salmonids. Fisheries Ecology and Management $19:$ 500-517.

Environment Agency., 2013. Managing water abstraction. Environment Agency, Bristol, UK : $29 \mathrm{p}$

European Commission., 2015. Ecological flows in the implementation of the Water Framework Directive. CIS Guidance Document No. 31. Technical Report $2015-086$ : $108 \mathrm{p}$.

Fahrner G., 2010. Typologie des impacts potentiels des ouvrages hydroélectriques sur les populations de truite situées en aval. Thèse de Doctorat. Agro Paris Tech, Paris : $168 \mathrm{p}$.

Floury C., Navarro L., Stroffek S., Dupré la Tour J. \& Lamouroux N., 2013. Mieux gérer les prélèvements d'eau : l'évaluation préalable des débits biologiques dans les cours d'eau. Note du Secrétariat Technique du Sdage. Agence de l'Eau Rhône-Méditerrannée-Corse, Irstea, Onema : $18 \mathrm{p}$.

Ghanem A. H., Steffler P.M., Hicks F.E. \& Katopodis C., 1996. Two dimensional finite element flow modeling of physical fish habitat. Regulated Rivers: Research and Management 12 : 185-200.

Gido K.B., Propst D.L., Olden J.D. \& Bestgen K.R., 2013. Multidecadal responses of native and introduced fishes to natural and altered flow regimes in the American Southwest. Canadian Journal of Fisheries and Aquatic Sciences 70 : 554-564.

Gillespie B.R., Desmet S., Kay P., Tillotson M.R. \& Brown L.E., 2015. A critical analysis of regulated river ecosystem responses to managed environmental flows from reservoirs. Freshwater Biology 60 : 410-425.

Ginot V., 1998. Logiciel EVHA. Evaluation de l'habitat physique des poissons en rivière (version 2.0). Cemagref Lyon $\mathrm{BEA} / \mathrm{LHQ}$ et Ministère de l'aménagement du Territoire et de l'Environnement, Direction de l'Eau, Paris : 109 p.

Girard V., Lamouroux N. \& Mons R., 2014a. Modeling point velocity and depth statistical distributions in steep tropical and alpine stream reaches. Water $R e$ sources Research 50 : 427-439.

Girard V., Monti D., Valade P., Lamouroux N., Mallet J.P. \& Grondin H., 2014b. Hydraulic preferences of shrimps and fishes in tropical insular rivers. River Research and Applications 30 : 766779.

Gouraud V., Capra H., Sabaton C., Tissot L., Vandewalle F., Lim P. \& Souchon Y., 2008. Long-term simulations of the dynamics of trout populations on river reaches bypassed by hydroelectric installations analysis of the impact of differents hydrological scénarios. River Research and Applications 24 : 11851205.

Hauer C., Unfer G., Holzapfel P., Haimann M. \& Habersack H., 2014. Impact of channel bar form and grain size variability on estimated stranding risk of juvenile brown trout during hydropeaking. Earth Surface Processes and Landforms 39 : 1622-1641.

Jowett I.G. \& Biggs B.J.F., 2006. Flow regime requirements and the biological effectiveness of habitat-based minimum 
flow assessments for six rivers. International Journal of River Basin Management 4 : 179-189.

Kendy E., Apse C. \& Blann K., 2012. A practical guide to environmental flows for policy and planning, with nine case studies from the United States. The Nature Conservancy, Arlington, VA (www.nature.org) : $74 \mathrm{p}$.

King J., Brown C. \& Sabet H., (2003) A scenario-based holistic approach to environmental flow assessments for rivers. River Research and Applications 19 : 619-639.

Lamouroux N., 2008. Hydraulic geometry of stream reaches and ecological implications. In Gravel Bed Rivers 6: From Process Understanding to the Restoration of Mountain Rivers. H. Habersack, H. Piégay and M. Rinaldi, ed. Developments in Earth Surface Processes 11 : 661-675.

Lamouroux N. \& Capra H., 2002. Simple predictions of instream habitat model outputs for target fish populations. Freshwater Biology 47 : 1543-1556.

Lamouroux N. \& Souchon Y., 2002. Simple predictions of instream habitat model outputs for fish habitat guilds in large streams. Freshwater Biology 47 : 15311542.

Lamouroux N. \& Olivier J.-M., 2015. Testing predictions of changes in fish abundance and community structure after flow restoration in four reaches of a large river (French Rhône). Freshwater Biology 60 : 1118-1130.

Lamouroux N., Capra H., Pouilly M. \& Souchon Y., 1999. Fish habitat preferences at the local scale in large streams of southern France. Freshwater Biology 42 : 673-687.

Lamouroux N., Mérigoux S., Capra H., Dolédec S., Jowett I.G. \& Statzner B.,
2010. The generality of abundanceenvironment relationships in microhabitats: a comment on Lancaster and Downes (2009). River Research and Applications 26 : 915-920.

Lamouroux N., Mérigoux S., Dolédec S. \& Snelder T.H., 2013. Transferability of hydraulic preference models for aquatic macroinvertebrates. River Research and Applications 29 : 933-937.

Lamouroux N., Pella H., Snelder T.H., Sauquet E., Lejot J. \& Shankar U., 2014. Uncertainty models for estimates of physical characteristics of river segments over large areas. Journal of the American Water Resources Association $50: 1-13$.

Lamouroux N., Gore J.A., Lepori F. \& Statzner B., 2015. The ecological restoration of large rivers needs sciencebased, predictive tools meeting public expectations: an overview of the Rhône project. Freshwater Biology 60 : 10691084.

Lancaster J. \& Downes B.J., 2010. Linking the hydraulic world of individual organisms to ecological processes: putting ecology into ecohydraulics. River Research and Applications 26 : 385-403.

Lang Delus C., 2011. Les étiages : définitions hydrologique, statistique et seuils réglementaires. Cybergeo : European Journal of Geography [www.cybergeo.revues.org/24827], Environnement, Nature, Paysage, document 571. DOI:10.4000/cybergeo.24827.

Linnansaari T., Monk W.A., Baird D.J. \& Curry R.A., 2013. Review of approaches and methods to assess Environmental Flows across Canada and internationally. Research Document 2012/039. Canadian Science Advisory Secretariat, Fisheries and Oceans, Canada : 75 p.

McManamay R.A., Orth D.J., Dolloff C.A. \& Mathews D.C., 2013. Application of the ELOHA framework to regulated rivers in 
the upper Tennessee river basin: a case study. Environmental Management 51 : 1210-1235.

Mérigoux S., Forcellini M., Dessaix J., Fruget J.-F., Lamouroux N. \& Statzner B., 2015. Testing predictions of changes in benthic invertebrate abundance and community structure after flow restoration in a large river (French Rhône). Freshwater Biology 60 : 1104-1117.

Miguel C., Lamouroux N., Pella H., Labarthe B., Flipo N., Akopian M. \& Belliard J., 2016. Altération d'habitat hydraulique à l'échelle des bassins versants: impacts des prélèvements en nappe du bassin Seine-Normandie. La Houille Blanche $3: 65-74$

Morandi B., Piégay H., Lamouroux N. \& Vaudor L., 2014. How is success or failure in river restoration projects evaluated? Feedback from French restoration projects. Journal of Environmental Management 137 : 178-188.

Murchie K.J., Hair K.P.E., Pullen A.C.E., Redpath A.T.D., Stephens A.H.R. \& Cooke S.J., 2008. Fish response to modified flow regimes in regulated rivers: research methods, effects and opportunities. River Research and Applications 24 : 197-217.

OldenJ.D., Konrad C.P., Melis T.S., Kennard M.J., Freeman M.C., Mims M.C., Bray E., Gido K., Hemphill N., Lytle D., McMullen L., Pyron M., Robinson C., Schmidt J. \& Williams J., 2014. Are large-scale flow experiments informing the science and management of freshwater ecosystems? Frontiers in Ecology and the Environment 12 : 176-185.

Pahl-Wostl C., Arthington A.H., Bogardi J., Bunn S.E., Hoff H., Lebel L., Nikitina E., Palmer M., Poff N. L., Richards K., Schluter M., Schulze R., St-Hilaire A., Tharme R., Tockner K. \& Tsegai D., 2013. Environmental flows and water governance: managing sustainable water uses. Current Opinion in Environmental Sustainability 5 : 341-351.

Pella H., Lejot J., Lamouroux N. \& Snelder $\mathrm{T}$., 2012. Le réseau hydrographique théorique (RHT) français et ses attributs environnementaux. Géomorphologie : Relief, Processus, Environnement 3 : 317-336.

Pelte T., Navarro L., Stroffek, Dupré la Tour J., Datry T., Langon M. Martinez P.-J. \& Delhaye H., 2014. Les cours d'eau intermittents. Eléments de connaissance et premières préconisations. Note du Secrétariat Technique du Sdage. Agence de l'Eau Rhône-MéditerrannéeCorse, Irstea, Onema, Dréal RhôneAlpes. Lyon, France : $16 \mathrm{p}$.

Poff N.L. \& Matthews J.H., 2013. Environmental flows in the Anthropocene: Past progress and future prospects. Current Opinion in Environmental Sustainability $5: 1-19$

Poff N.L. \& Zimmerman J.K.H., 2010. Ecological responses to altered flow regimes: a literature review to inform the science and management of environmental flows. Freshwater Biology 55 : 194-205.

Poff N.L., Allan J.D., Bain M.B., Karr J.R., Prestegaard K.L., Richter B.D., Sparks R., \& Stromberg J., 1997. The natural flow regime. A paradigm for river conservation and restoration. BioScience 47 : 769-784.

Poff N.L., Richter B.D., Arthington A.H., Bunn S.E., Naiman R.J., Kendy E., Acreman M.C., Apse C., Bledsoe B.P., Freeman M.C., Henriksen J., Jacobson R.B., Kennen J.G., Merritt D.M., O'Keeffe J.H., Olden J.D., Rogers K., Tharme R.E. \& Warner A., 2010. The ecological limits of hydrologic alteration (ELOHA): a new framework for developing regional environmental flow standards. Freshwater Biology 55 : 147-170. 
République Française., 2011. Circulaire du 5 Juillet 2011 relative à l'application de l'article L. 2014-18 du code de l'environnement sur les débits réservés à maintenir en cours d'eau. Ministère de l'Ecologie, du Développement Durable, des Transports et du Logement : $5 \mathrm{p}$.

Richter B.D., 2010. Re-thinking environmental flows: from allocations and reserves to sustainability boundaries. River Research and Applications 26 : 1052-1063.

Richter B.D., Baumgartner J.V., Powell J. \& Braun D.P., 1996. A method for assessing hydrologic alteration within ecosystems. Conservation Biology 10 : 11631174.

Richter B.D., Baumgartner J.V., Wigington R. \& Braun D.P., 1997. How much water does a river need? Freshwater Biology 37 : 231-249.

Riffard M., Andréassian V., Nicolle P. \& Peschard J., 2011. Combinaison multimodèle et cartographie de consensus du débit de référence d'étiage et du débit moyen à l'échelle de la France. Rapport technique. Onema \& Irstea, Vincennes, France : $37 \mathrm{p}$.

Rinaldi M., Belletti B., Van de Bund W., Bertoldi W., Gurnell A., Buijse T. \& Mosselman E., 2013. Review on ecohydromorphological methods. In Deliverables of the EU FP7 REFORM project. N. Friberg, M. O'Hare and A. Poulsen ed. (www.reformrivers.eu) : 202 p.

Rolls R.J., Leigh C. \& Sheldon F., 2012. Mechanistic effects of low-flow hydrology on riverine ecosystems:ecological principles and consequences of alteration. Freshwater Science 31 : 11631186.

Sabaton C., Valentin S. \& Souchon Y., 1995. La méthode des microhabitats. Protocoles d'application. EDF Direction des Etudes et Recherches et Cemagref : $32 \mathrm{p}$.
Sabaton C., Souchon Y., Capra H., Gouraud V., Lascaux J.-M. \& Tissot L., 2008. Long-term brown trout populations responses to flow manipulation. River Research and Applications 24 : 476505.

Saraeva K. \& Hardy T.B., 2009. Prediction of fisheries physical habitat values based on hydraulic geometry and frequency distributions of depth and velocity. International Journal of River Basin Management 7 : 31-41.

Sauquet E. 2006. Mapping mean annual river discharges: geostatistical developments for incorporating river network dependencies. Journal of Hydrology 331(1-2) : 300-314.

Sauquet E. \& Catalogne C. 2011. Comparison of catchment grouping methods for flow duration curve estimation at ungauged sites in France. Hydrology and Earth System Sciences 15 : 24212435.

Shenton W., Bond N.R., Yen J.D.L. \& Mac Nally R., 2012. Putting the "Ecology" into environmental flows: ecological dynamics and demographic modelling. Environmental Management 50 : 1-10.

Singh V. P. \& Frevert D.K., 2006. Watershed Models. CRC Press, Boca Raton, Florida, USA : $653 \mathrm{p}$.

Snelder T.H. \& Lamouroux N., 2010. Covariation of fish assemblages, flow regimes and other habitat factors in French rivers. Freshwater Biology 55 : 881-892.

Snelder T.H., Lamouroux N., Leathwick J.R., Pella H., Sauquet E. \& Shankar U., 2009. Predictive mapping of natural flow regimes of France. Journal of Hydrology $373: 57-67$.

Snelder T.H., Booker D. \& Lamouroux N., 2011. A method to assess and define environmental flow rules for large jurisdictional regions. Journal of the American Water Resources Association 47 : 828-840. 
Snelder T.H., Datry T., Lamouroux N., Larned S.T., Sauquet E., Pella H. \& Catalogne C., 2013. Regionalization of patterns of flow intermittence from gauging station records. Hydrology and Earth System Sciences 17 : 2685-2699.

Souchon Y., Lamouroux N., Capra H. \& Chandesris A., 2003. La méthodologie Estimhab dans le paysage des méthodes de microhabitat. Note technique, Cemagref Lyon: 9 pp. (http:// www.irstea.fr/dynam).

Souchon Y., Sabaton C., Deibel R., Reiser D., Kershner J., Gard M., Katopodis C., Leonard P., Poff N.L., Miller W.J. \& Lamb B.L., 2008. Detecting biological responses to flow management: missed opportunities; future directions. River Research and Applications 24 : 506-518.

Statzner B. \& Müller R., 1989. Standard hemispheres as indicators of flow characteristics in lotic benthos research. Freshwater Biology 21 : 445-459.

Tennant D.L., 1976. Instream flow regimens for fish, wildlife, recreation and related environmental resources. Fisheries 1 : 6-10.

Tharme R.E., 2003. A global perspective on environmental flow assessment: emerging trends in the development and application of environmental flow methodologies for rivers. River $R e$ search and Applications 19 : 397-441.

Thévenet A., 1998. Intérêt des débris ligneux grossiers pour les poissons dans les grandes rivières. Thèse de doctorat, Université Lyon I, Lyon, France : $111 \mathrm{p}$.

Valette L., Piffady J., Chandesris A. \& Souchon Y., 2012. SYRAH-CE: description des données et modélisation du risque d'altération hydromorphologique des cours d'eau pour l'état des lieux DCE. Rapport Technique Onema-Irstea: $104 \mathrm{p}$.

Vaudor L., Lamouroux N., Olivier J.-M. \& Forcellini M., 2015. How sampling influences the statistical power to detect changes in abundance: an application to river restoration. Freshwater Biology $60: 1192-1207$.

Webb J.A., De Little S.C., Miller K.A., Stewardson M.J., Rutherfurd I.D., Sharpe A.K., Patulny L. \& Poff N.L., 2015. A general approach to predicting ecological responses to environmental flows: making best use of the literature, expert knowledge and monitoring data. River Research and Applications 31 : 505-514.

Wilding T.K., Bledsoe B., Poff N.L. \& Sanderson J., 2014. Predicting habitat response to flow using generalized habitat models for trout in Rocky Mountain streams. River Research and Applications 7 : 805-824. 